\title{
Combination of Microneedling and 10\% Trichloro-Acetic Acid Peeling Versus Platelet Rich Plasma in the Treatment of Infraorbital Dark Circles
}

\author{
A.I.El-Taweel, A.I.Mustafa and I.Q.Qiresh
}

Dermatology, Venereology and Andrology Dept., Faculty of Medicine, Benha Univ., Benha, Egypt

E-Mail:Ikram@gmail.com

\begin{abstract}
Dark circles are a common benign facial cosmetic problem with multiple factorial etiologies. Though, there are a number of treatment options available for periorbital hyperpigmentation. It can be a significant cosmetic problem, and many individuals try to find a treatment for this condition. This condition affects individuals in a wide range of ages, both sexes and all races. Moreover, it worsens with the aging and the process of skin sagging and altered subcutaneous fat distribution. Assess those viability and safety about consolidation for microneedling What's more $10 \%$ trichloroacetic corrosive peeling Furthermore thinking about it with platelet rich plasma intradermal infusion in the medicine from claiming infraorbital dim circles. 60 members finished those study; assembly (a), included 30 patients were aPProached with microneedle help Furthermore topical anesthesia requisition from claiming trichloracetic corrosive $10 \%$ answer for each infraorbital range for 5 minutes. Compound peeling might have been performed each 2 weeks for four treatments; assembly (b), included 30 patients were treated for intradermal platelet rich plasma. Microneedle and PRP gatherings were connected with change for dull circles. Superior reaction What's more patients' fulfillment were fundamentally connected with microneedle At contrasted with PRP. Patients fulfillment might have been essentially connected with change. We inferred that those two routines for medicine were just as powerful in the medication of DC, with those change from claiming dc watched from the principal medication session for both Microneedling with compound peeling Eventually Tom's perusing 10\% TCA Furthermore PRP infusion medication.
\end{abstract}

Keywords: Infraorbital Dark Circles, Micro needling; Platelet Rich Plasma, Trichloro-Acetic Acid.

\section{Introduction}

Infraorbital dull circis siliquastrum alludes to states that introduce for relative haziness of the infraorbital eyelids. It might a chance to be a huge cosmea problem, Furthermore Numerous people look for medicine to this condition, in any case there have been few investigations viewing those foundation What's more little research under the possibility medication for this state. This state influences people with an extensive variety for age, both sexes What's more every last bit races. Moreover, it worsens with the agincourt methodology from claiming skin drooping What's more modified subcutaneous fat conveyance [1].

Cosmea states that are not wellbeing not debilitating or connected with critical horribleness At that could influence the individual's passionate prosperity would picking up expanded consideration. Infraorbital dim circles are An state that might a chance to be An critical cosmea worry for female patients. Those etiology about periocular pigmentation might make multifactorial with no etiologic agenize predominating. Could reasonably be expected causative Components of the dim circles includeexcessivepigmentation; thin, translucent bring down eyelidskin overlying theorbicularis oculi muscle; Furthermore shadowing because of skin laxity What's more shred trounce [2].

To general, medicine for depigmenting operators must make proceeded to a few months preceding cosmea reductions need aid gotten. The movement instruments from claiming depigmenting operators are restraint of tyrosinase activity, restraint about dna union On hyperactive melanocytes, diminishment of epidermal content from claiming melanin, Furthermore thickening of the epidermis (granular layer) [2].
Same time concoction peeling need been used to treat an assortment of facial Pigmentary disorders, there would no randomized-control investigations on the viability of compound peeling to those medication about periocular hyperpigmentation. Those peeling operators uproot melanin starting with those stratum corneum Also epidermis. Profound peels might uproot melanin starting with dermis Yet might prompt dyspigmentation What's more scarring. In this areathe skin will be thin, thus profound peels need aid not proposed [3].

Lasers bring progressively been utilized within cosmea dermatology. Dull circles because of unreasonable pigmentation need been effectively treated with Different pigment lasers [4].

An additional reason for infraorbital dull circles may be dainty and translucent easier eyelid skin overlying those orbicularis oculi muscle. This hyper vascular manifestation may be because of a mix about expanded vascularity of the subcutaneous vascular plexus or vasculature held inside the muscle Furthermore transparency of the overlying skin. The restorative modality for these instances will be should restore the volume underneath the eyelid utilizing autologous fat transplantation or delicate tissue fillers. Autologous fat transplantation need in length been utilized for soft-tissue growth [5].

Medicine for infraorbital dim circles identified with shred trounce will be that's only the tip of the iceberg perplexing. It might oblige obtrusive surgical methods with lift those delicate tissues starting with those underlying maxilla, fat transplantation or fat extrusion, and septal resection [5].

The point of the exhibit consider might have been with assess the viability Furthermore wellbeing of blending for microneedling Also 10\% trichloro acidic 
corrosive peeling Furthermore contrasting it for platelet rich plasma intradermal infusion in the medication of infraorbital dim circles.

\section{Patients and methods}

This cross-sectional study incorporated 60 patients torment starting with dim circles. Patients were chose starting with patients going to dermatology What's more Andrologyout-patient facility for Benha school healing centers. Starting with the period from claiming might 2017 till november 2018. Know patients gained complete majority of the data once steps from claiming medication and various medication sessions were important for fulfillment.

Those contemplate might have been affirmed Toward the nearby morals panel around Scrutinize directing, including mankind's subjects for Benha employees for pharmaceutical. A educated assent might have been gotten from each distinct in the recent past being selected in the consider. Patients were arranged under two aggregations. Bunch A: 30 Patients were dealt with with programmed microneedle treatment Dermapen (system-handhold AMTS-H, Mcure, Co, Ltd) and topical anesthesia provision about trichloracetic corrosive $10 \%$ answer for each infraorbital territory to 5 minutes. Concoction peeling were performed each 2 weeks for four medicines. The impact were photograph archived and a tolerant Furthermore doctor worldwide evaluation might have been assessed. Security might have been evaluated Toward assessing punctual What's more deferred unfriendly occasions. Assembly B: 30 Patients were treated with intradermal platelet rich plasma. The medicine span might have been comprise of three sessions from claiming autologous platelet-rich. Plasma injections under those periorbital region with particular case month separated amidst those injections Also trailed up to extra three months to assess those level of change alternately repeat of the dull circles.

\section{Inclusion criteria}

Patients with mild to severe infraorbital dark circles.

\section{Exclusion criteria}

- Patients with known positive history of keloidal tendency.

- Patients with positive history of bleeding or platelet disorder.

Table (1) Baseline data among the studied groups
- Patients with positive history of major surgery in past 6 months.

- Presence of any acute infection on face like, herpes, folliculitis.

- Patients with HIV, HBsAg, or any chronic illness.

- Pregnancy.

All patients were subjected to complete history was taken from each patient, complete general and dermatological clinical examination was done, and Clinical details of all patients were recorded.

\subsection{Statistical methods}

Those gathered information might have been revised, coded, tabulated and presented on a pc utilizing Factual bundle to social science (IBM corp. Discharged 2011. IBM SPSS facts for Windows, rendition 20. 0. Armonk, NY: IBM corp. ). Information were introduced Furthermore suitableness examination might have been carried as stated by the sort about information acquired for each parameter. Spellbinding statistics: intend standard deviation ( \pm SD) to parametric numerical information. Recurrence Also rate of non-numerical information. Explanatory statistics: scholar $\mathrm{t}$ test might have been used to evaluate those Factual essentialness of the Contrast between two examine gathering intends. Chi-Square test might have been used to inspect those association between two qualitative variables. Fisher's correct test: might have been used to inspect those association between two qualitative variables when those normal check will be less 5 for more than $20 \%$ about phones. Relationship analysis: Pearson's connection might have been used to evaluate those quality for companionship between two quantitative variables. Those relationship coefficient characterizes the quality Also bearing of the straight association between two variables. Relapse analysis: straight relapse dissection might have been utilized to prediction for change.

\section{Results}

The present study was conducted on 60 female patients with dark circles. Their mean age was 31.1 years. Most cases suffered of excess stress $(81.7 \%)$. Family history was positive in $48.3 \%$. Most cases were free of systemic diseases $(96.7 \%)$. Only 1 was diabetic and 1 was hypertensive.

No significant differences were found regarding age, stress, family history, history of systemic diseases, and treatment between studied groupsTable (1).

\begin{tabular}{|c|c|c|c|c|}
\hline & & $\begin{array}{c}\text { Microneedle } \\
\mathbf{N}=30\end{array}$ & $\begin{array}{c}\text { PRP } \\
\mathbf{N}=30\end{array}$ & $p$ \\
\hline Age (years) & $\begin{array}{c}\text { Mean } \\
\pm \text { SD }\end{array}$ & $\begin{array}{c}33 \\
\pm 9.3\end{array}$ & $\begin{array}{l}31.2 \\
\pm 9.1\end{array}$ & 0.139 \\
\hline Excess stress & $\begin{array}{l}\mathbf{N} \\
\%\end{array}$ & $\begin{array}{c}26 \\
86.7 \%\end{array}$ & $\begin{array}{c}23 \\
76.7 \%\end{array}$ & 0.317 \\
\hline Positive family history & $\begin{array}{l}\mathbf{N} \\
\%\end{array}$ & $\begin{array}{c}15 \\
50 \%\end{array}$ & $\begin{array}{c}14 \\
46.7 \%\end{array}$ & 0.796 \\
\hline
\end{tabular}


Table (3) Continue

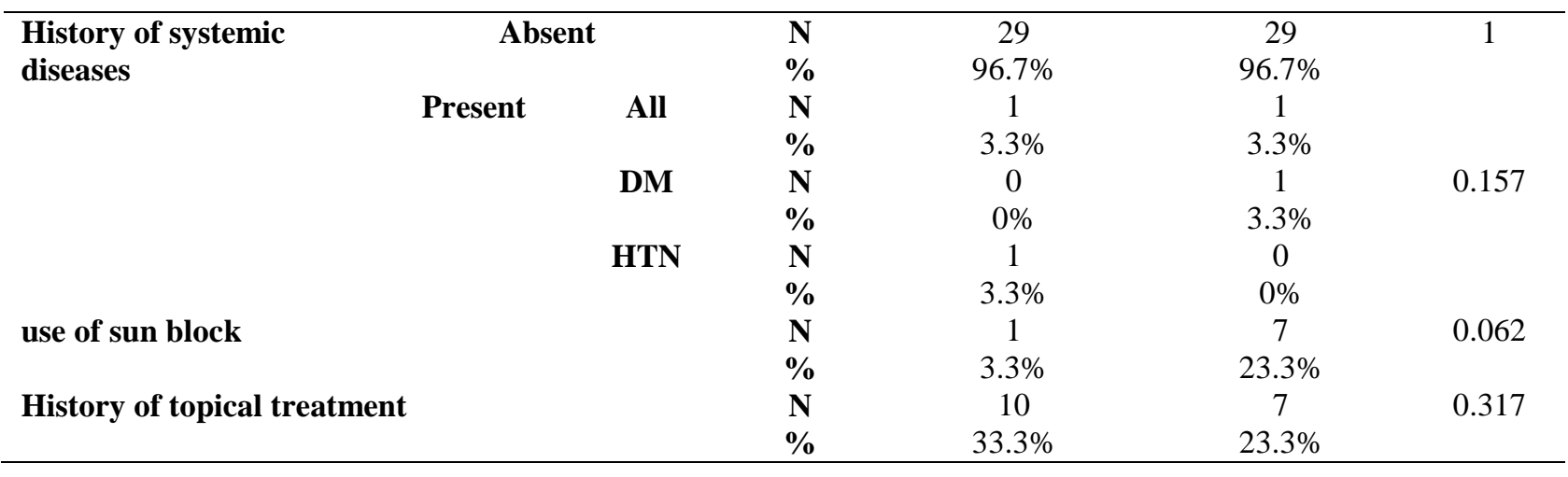

No significant differences were found regarding grade, onset, course, duration between studied groups Table (2).

Table (2) Dark circles' features of all studied groups.

\begin{tabular}{|c|c|c|c|c|c|c|c|}
\hline \multirow{3}{*}{ Grade } & \multirow[b]{3}{*}{ I } & \multirow[b]{3}{*}{$\mathrm{N}, \%$} & \multirow{2}{*}{\multicolumn{2}{|c|}{$\begin{array}{c}\text { Microneedle } \\
\mathbf{N}=\mathbf{3 0} \\
\end{array}$}} & \multirow{2}{*}{\multicolumn{2}{|c|}{$\begin{array}{c}\text { PRP } \\
\mathbf{N}=30\end{array}$}} & \multirow[t]{2}{*}{$\mathbf{p}$} \\
\hline & & & & & & & \\
\hline & & & 1 & $3.3 \%$ & 1 & $3.3 \%$ & \multirow[t]{4}{*}{0.444} \\
\hline & II & $\mathrm{N}, \%$ & 19 & $63.3 \%$ & 13 & $43.3 \%$ & \\
\hline & III & $\mathrm{N}, \%$ & 8 & $26.7 \%$ & 13 & $43.3 \%$ & \\
\hline & IV & $\mathrm{N}, \%$ & 2 & $6.7 \%$ & 3 & $10 \%$ & \\
\hline \multirow[t]{2}{*}{ Onset } & Gradual & $\mathrm{N}, \%$ & 27 & $90 \%$ & 26 & $86.7 \%$ & \multirow[t]{2}{*}{0.688} \\
\hline & Sudden & $\mathrm{N}, \%$ & 3 & $10 \%$ & 4 & $13.3 \%$ & \\
\hline \multirow[t]{2}{*}{ Course } & Stationary & $\mathrm{N}, \%$ & 8 & $26.7 \%$ & 4 & $13.3 \%$ & \multirow[t]{2}{*}{0.197} \\
\hline & Progressive & $\mathrm{N}, \%$ & 22 & $73.3 \%$ & 26 & $86.7 \%$ & \\
\hline Duration (years) & & Mean \pm SD & 3 & \pm 0.9 & 2.5 & \pm 0.7 & 0.118 \\
\hline
\end{tabular}

Better response was significantly associated with group A when compared to group B ( $\mathrm{p}=0.041)$ Table (3).

Table (3) Response among all studied groups.

\begin{tabular}{|c|c|c|c|c|c|c|}
\hline & & \multirow{2}{*}{\multicolumn{2}{|c|}{$\begin{array}{c}\text { Microneedle } \\
\mathbf{N}=\mathbf{3 0}\end{array}$}} & \multirow{2}{*}{\multicolumn{2}{|c|}{$\begin{array}{c}\text { PRP } \\
\mathbf{N}=\mathbf{3 0}\end{array}$}} & \multirow[t]{3}{*}{ p } \\
\hline & & & & & & \\
\hline & & $\mathbf{N}$ & $\%$ & $\mathbf{N}$ & $\%$ & \\
\hline \multirow[t]{4}{*}{ Response } & poor & 5 & $16.7 \%$ & 15 & $50 \%$ & 0.041 \\
\hline & fair & 8 & $26.7 \%$ & 7 & $23.3 \%$ & \\
\hline & good & 11 & $36.7 \%$ & 6 & $20 \%$ & \\
\hline & excellent & 6 & $20 \%$ & 2 & $6.7 \%$ & \\
\hline
\end{tabular}

Frequency of unbearable pain was associated with group B when compared to group A, although did not reach significant level ( $\mathrm{p}>0.05)$ Table (4).

Table (4) Pain assessment in all studied groups

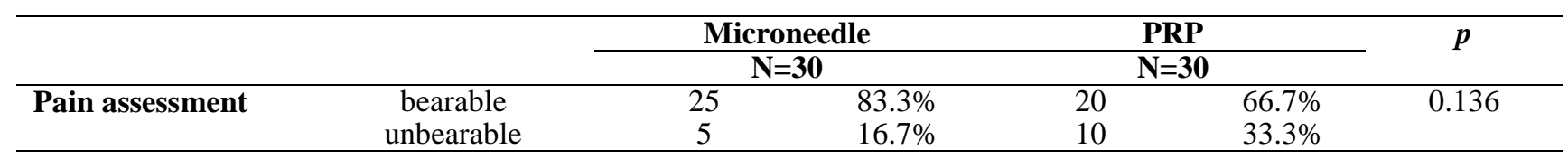

No significant differences were found regarding side effects between studied groups Table (5).

Table (5) Side effects in all studied groups.

\begin{tabular}{|c|c|c|c|c|c|c|}
\hline & & & edle & & & $p$ \\
\hline Side effects & $\begin{array}{l}\text { Absent } \\
\text { hyperpigmented spot }\end{array}$ & $\begin{array}{l}29 \\
1\end{array}$ & $\begin{array}{l}96.7 \% \\
3.3 \%\end{array}$ & $\begin{array}{l}29 \\
1\end{array}$ & $\begin{array}{l}96.7 \% \\
3.3 \%\end{array}$ & 1 \\
\hline
\end{tabular}




\section{Discussion}

Microneedling otherwise called collagen incitement help is An basic office-based system. The medicinal dermaroller needles penetrate the stratum corneum What's more make micro-conduits without harming the epidermis. On an epithelial micro-injury occurs, those skin phones discharge potassium What's more proteins that as an exchange change the conductivity of the interstitium. In the same time, those internal cell division possibility increments dramatically on -120 Also more mv. This possibility distinction strengths fibroblast on move keeping to the point from claiming damage and At last powers them will proliferate [6].

This methodology prompts the arrival for Growth factors prompting those framing from claiming new collagen Also elastin in the papillary dermis [7].

In the available investigation compound peels for $10 \%$ TCA acted Similarly as an adjuvant with microneedling, as it reasons controlled skin damage, pushing recovery Furthermore revival for tissues [8].

TCA could achieve the dermis utilizing An lowconcentration result connected should a epidermis that need been a greater amount penetrability Eventually Tom's perusing microneedling. Infiltration for TCA particles is quick due to its really lipophilic way. It might turned a medium-depth peel contingent upon the number from claiming layers connected in the same session, those contact time, the amount from claiming medication sessions formerly performed, those time interim the middle of them and the skin thickness of the treated region [9].

On addition, those skin of the infraorbital eyelids may be extremely slim (thickness of roughly $0.5 \mathrm{~mm}$ ). TCA fortifies neocollagenesis, produces scattering from claiming melanin Furthermore enhances At whatever post-inflammatory hyperpigmentation brought on by microneedling itself. As stated by those effects of the contemplate both those routines suPPlement one another to provide for fantastic cosmea brings about those decrease for pigmentation On dim circles.

Our consider might have been performed around 60 diverse skin Characteristics cases, best $6.7 \%$ about them required melasma, skin sort i might have been display clinched alongside $1.7 \%$, sort ii for $15 \%$, kind iii for 68 . $3 \%$ Also kind iv clinched alongside $15 \%$. Distinctive evaluations of dim circles were evaluated to them, 3. 3\% required review I, 53. 3\% required review II, 35\% needed evaluation iii What's more $8.3 \%$ needed evaluation iv. No huge contrasts were found in regards to age, stress, gang history, historical backdrop about systemic diseases, skin kind What's more melisma Also medicine between contemplated Assemblies. Onset might have been gradual over 88 . $3 \%$ What's more sudden passing over $11.7 \%$. Course might have been stationary Previously, 20\% Furthermore progressive in $80 \%$ for a imply span might have been 3 . 2 quite some time. No huge contrasts were found in regards to onset, course What's more span. There might have been huge change done gathering (A) who's aPProached for microneedling Also $10 \%$ trichloroacetic corrosive peels. This might have been in understanding for Kontochistopoulos et al; [10]. Our consider indicated phenomenal change in $20 \%$ about instances of assembly (A),. Platelet-rich plasma may be an autologous centralization for human platelets for a little volume from claiming plasma. When the platelets are actuated Toward calcium chloride, thrombin alternately fibrinogen, they discharge their Growth Components [11].

Platelets hold numerous and emit seven diverse Growth Components being PDGFaa, PDGFab and PDGFbb, TGF b1, TGF b2, VEGF and EGF alongside fibrin, fibronectin Also vibronectin, which assume separate parts in wound recuperating Furthermore cell adhesion, Likewise An grid to movement in distinctive tissues including bone, connective tissue, What's more epithelium. Correct PRP will be constantly autologous, not homologous. PRP worth of effort will be through the degranulation of platelets' An granules holding put away Growth elements. Platelet discharge alternately fixation of development variables done PRP relies on the gadget utilized with which platelets need aid actuated [12].

Receptors for development Components clinched alongside PRP are found ahead grown-up mesenchymal stem cells, fibroblasts, osteoblasts, endothelial cells, and epidermal cells, on get ready PRP, centrifugation about blood proceeds until it separates under three layers continuously red platelets (RBCs), platelet-poor plasma (PPP) Furthermore PRP. Usually, this takes spot over two sequential steps; In PPP is divided Also then, RBCs is differentiated starting with PRP. PRP might afterward make differentiated from different parts Also connected [13].

PRP stays sterile Also amassed platelets remain feasible dependent upon $8 \mathrm{~h}$ whether they would produced clinched alongside anticoagulated state Also set once a sterile surgical table. The focal point of autologous exchange may be keeping those transmission about micro-organisms for example, HIV, hepatitis viruses, and so forth throughout this way, observing and stock arrangement of all instrumentation may be enha. [13].

Platelet-rich plasma need the possibility should go about as a stimulant to fibroblast burgeoning What's more collagen arrival done tissue societies. There may be an absence about target histological proof from claiming neocollagenesis fortified Toward PRP [11].

For An investigation utilizing platelet-rich fibrin grid to moderate-to-severe nasolabial folds to 15 members accompanied dependent upon to 12 weeks, investigators accounted a critical change clinched alongside wrinkle aPPraisal scores $(\mathrm{P}<0.001)$ [14].

We didn't examine nasolabial folds. However, our analyses once Possibly side from claiming infra-orbital territories preferably in detached skin of the bring 
down cover Furthermore contiguous skin with respect to each side indicated fantastic reaction to just $6.7 \%$ of aggregation (B).

For na et al; [15] study, melanin list Also erythema list were compared between two sides. Person side underwent ablative co2 fragmentary laser performed on inward surface of the arm; ordinary saline might have been utilized as a control with PRP. Alternate side underwent ablative co2 fragmentary laser treatment Besides PRP. Melanin list Furthermore erythema list were both fundamentally lessened on the side, which accepted PRP $(\mathrm{P}<0.05)$.

On the different hand, Yun et al; [16] investigated the part of EGF On melanogenesis by quest for outflow about EGFR for melanocytes and the impact from claiming EGF ahead melanin generation Toward melanocytes with alternately without laser-treated keratinocyte-conditioned society networking. Melanocytes treated for laser-treated keratinocyteconditioned society networking needed more terrific prostaglandin-E2 (PGE2) outflow Furthermore tyrosinase catalyst action over melanocytes treated with control media, Also medication with EGF brought down melanin handling of melanocytes about lasertreated keratinocyte-conditioned society networking Yet not from claiming melanocytes treated for control networking. So, it Might make contended that some Growth factors, for example, those two, might decrease those union of melanin through tyrosinase or some other instruments over melanogenesis.

So, PRP Similarly as a intense hotspot of development factors, might make seen Likewise a safe, biocompatible, autologous Also proper medication modality to the dull eye circles structured done more level eye locales which need An slim skin that camwood expand the caliber of patients' exists As far as social Furthermore mental capacity.

\section{Conclusion}

We concluded that the two methods of treatment were equally effective in the treatment of DC, with the improvement of DC observed from the first treatment session for both Microneedling with chemical peeling by $10 \%$ TCA and PRP injection treatment. Microneedling with chemical peeling TCA $10 \%$ was much better in the treatment of DC, with minimal tolerated side effects. It is cheap and is considered as a simple in-office technique. On the other hand, PRP injection as a potent source of growth factors can be seen as a safe, biocompatible, autologous and aPPropriate treatment modality for the dark eye circles formed in lower eye regions which has a thin skin that can increase the quality of patients' lives in terms of social and psychological function.

\section{References}

[1] M. Yaar , B. A. Gilchrest, "Skin aging: postulated mechanisms and consequent changes in structure and function," Clin. Geriatr. Med, Vol.17(4), PP. 617-630, 2001.
[2] M. R. Roh , K. Y. Chung, "Infraorbital dark circles: definition, causes, and treatment options," Dermatologic Surg, Vol.35(8), PP. 1163-1171, 2009.

[3] H. Al-Talib, A. Al-Khateeb, A. Hameed, C. Murugaiah, "Efficacy and safety of superficial chemical peeling in treatment of active acne vulgaris," An. Bras. Dermatol, Vol.92(2), PP. 212-216, 2017.

[4] R. Sarkar, R. Ranjan, S. Garg, V. K. Garg, S. Sonthalia, S. Bansal, "Periorbital hyperpigmentation: a comprehensive review," J. Clin. Aesthet. Dermatol, Vol.9(1), PP. 49, 2016.

[5] I. Vrcek, O. Ozgur, T. Nakra, "Infraorbital dark circles: A review of the pathogenesis, evaluation and treatment," J. Cutan. Aesthet. Surg, Vol.9(2), PP. 65, 2016.

[6] G. Fabbrocini, N. Fardella, A. Monfrecola, I. Proietti, D. Innocenzi, "Acne scarring treatment using skin needling," Clin. Exp. Dermatology Exp. dermatology, Vol.34(8), PP. 874-879, 2009.

[7] J. Sharad, "Combination of microneedling and glycolic acid peels for the treatment of acne scars in dark skin," J. Cosmet. Dermatol, Vol.10(4), PP. 317-323, 2011.

[8] M. Landau, R. Wolf, N. Curcio, "Cosmetic complications," Emerg. Dermatology; vol.1(10), PP. 323, 2017.

[9] C. Vavouli , "Chemical peeling with trichloroacetic acid and lactic acid for infraorbital dark circles," J. Cosmet. Dermatol, Vol.12(3), PP. 204-209, 2013.

[10] G. Kontochristopoulos, A. Kouris, E. Platsidaki, V. Markantoni, M. Gerodimou, C. Antoniou, "Combination of microneedling and $10 \%$ trichloroacetic acid peels in the management of infraorbital dark circles," J. Cosmet. Laser Ther, Vol.18(5), PP. 289-292, 2016.

[11]D. F. du Toit, W. G. Kleintjes, J. Morkel, E. J. Mazyala, B. J. Page, "Soft and hard-tissue augmentation with platelet-rich plasma: Tissue culture dynamics, regeneration and molecular biology perspective.," Int. J. Shoulder Surg, Vol.1(2),PP.58-65, 2007.

[12]L. Mazzucco, V. Balbo, E. Cattana, P. Borzini, "Platelet-rich plasma and platelet gel preparation using Plateltex ${ }^{\circledR}, "$ Vox Sang, Vol.94(3), PP. 202208, 2008.

[13] H.-L. Wang , G. Avila, "Platelet rich plasma: myth or reality?," Eur. J. Dent, Vol.7(1), PP. 192-194, 2013.

[14]A. P. Sclafani, "Platelet-rich fibrin matrix for improvement of deep nasolabial folds," J. Cosmet. Dermatol, Vol.9(1), PP. 66-71, 2010.

[15] J. NA , "Rapid healing and reduced erythema after ablative fractional carbon dioxide laser resurfacing combined with the aPPlication of autologous platelet-rich plasma," Dermatologic Surg, Vol.37(4), PP. 463-468, 2011.

[16] W. J. Yun, S. H. Bang, K. H. Min, S. W. Kim, M. 
W. Lee, S. E. Chang, "Epidermal growth factor and epidermal growth factor signaling attenuate laser-induced melanogenesis," Dermatologic Surg, Vol.39(12), PP. 1903-1911, 2013. 